\title{
Identification of Intracellular $\beta$-Barrel Residues Involved in Ion Selectivity in the Mechanosensitive Channel of Thermoanaerobacter tengcongensis
}

\author{
Yingcai Song ${ }^{1 \dagger}$, Bing Zhang ${ }^{1,2 \dagger}$, Fei Guo ${ }^{2 \dagger}$, Maojun Yang ${ }^{3 *}$, Yang $\mathrm{Li}^{2 *}$ and Zhi-Qiang Liu ${ }^{1 *}$ \\ ${ }^{1}$ Department of Anaesthesiology, Shanghai First Maternity and Infant Hospital, Tongji University School of Medicine, \\ Shanghai, China, ${ }^{2}$ Key Laboratory of Receptor Research, Shanghai Institute of Materia Medica, Chinese Academy of \\ Sciences, Shanghai, China, ${ }^{3}$ Key Laboratory for Protein Sciences of Ministry of Education, Tsinghua-Peking Center for Life \\ Sciences, School of Life Sciences, Tsinghua University, Beijing, China
}

OPEN ACCESS

Edited by:

Christoph Fahlke,

Forschungszentrum Jülich, Germany

Reviewed by:

Delany Torres-Salazar

National Institutes of Health (NIH),

United States

Marcelo Catalan,

Arturo Prat University, Chile

*Correspondence:

Maojun Yang

maojunyang@tsinghua.edu.cn

Yang Li

liyang@simm.ac.cn

Zhi-Qiang Liu

drliuzhq@hotmail.com

${ }^{\dagger}$ These authors have contributed equally to this work.

Specialty section:

This article was submitted to Membrane Physiology and Membrane Biophysics,

a section of the journal

Frontiers in Physiology

Received: 19 June 2017 Accepted: 06 October 2017 Published: 25 October 2017

Citation:

Song $Y$, Zhang $B$, Guo $F$, Yang $M$, Li Y and Liu Z-Q (2017) Identification of Intracellular $\beta$-Barrel Residues

Involved in lon Selectivity in the

Mechanosensitive Channel of Thermoanaerobacter tengcongensis.

Front. Physiol. 8:832

doi: 10.3389/fphys.2017.00832
The mechanosensitive channel of small conductance (MscS) is a bacterial membrane pore that senses membrane tension and protects cells from lysis by releasing osmolytes. MscS is a homoheptameric channel with a cytoplasmic domain with seven portals and a $\beta$-barrel opening to the cytoplasm. TtMscS, an MscS channel from Thermoanaerobacter tengcongensis, is an anion-selective channel. A previous study from our laboratory has defined the crucial role of $\beta$-barrel in the anion selectivity of TtMscS (Zhang et al., 2012). However, the mechanistic details by which the $\beta$-barrel determines anion selectivity remain unclear. Here, using mutagenesis and patch-clamp recordings, we investigated the function and structural correlations between $\beta$-barrels and the anion selectivity of TtMscS at the atomic level. Our results indicated that mutation of $\mathrm{V} 274$, a residue at the center of the inner wall of the $\beta$-barrel in TtMscS, caused the anion selectivity of TtMscS reverse to cation selectivity. Moreover, the electrostatic potential (T272) and physical size ( $L 276)$ of residues in the inner wall of $\beta$-barrel also determine the anion selectivity of TtMscS. In summary, the present study confirmed that the $\beta$-barrel region of TtMscS acts as a "selective filter" that renders TtMscS anion selectivity.

Keywords: TtMscS, anion selectivity, $\beta$-barrel, selective filter, patch-clamp, single mutation

\section{INTRODUCTION}

Mechanosensitive (MS) channels are membrane pores that are universally present in prokaryotes and eukaryotes (Martinac et al., 2008; Kung et al., 2010). These channels sense membrane tension and consequently accelerate the passage of osmolytes from areas of high concentration to those of low concentration (Kung, 2005). In bacteria, MS channels can be activated by turgor pressure. Like an "emergency valve," when bacteria experience hypoosmotic shock, such as during rain, the channels open and release osmolytes along with water, thus alleviating cell turgor and protecting cells from lysis (Martinac et al., 1987). Additionally, a recent study has suggested that MS channels play a crucial role in the calcium regulation of bacteria with life-threatening hypoosmotic conditions (Cox et al., 2013), thereby ensuring bacterial survival across a wide range of external osmolarity levels. In mammals, MS channels have been implicated in numerous physiological processes, including touch, pain sensation, hearing, blood pressure control, micturition, tissue growth, cell volume regulation, and turgor control (Arnadóttir and Chalfie, 2010; Ranade et al., 2015). 
The mechanosensitive channel of small conductance (MscS) is a homoheptameric channel that belongs to a family of pressuresensitive channels. MscS channels are small conductance channels that open at a slight pressure of tens of $\mathrm{mmHg}$; this pressure may result from an osmotic difference of only a few milliosmoles through the membrane (Martinac et al., 1987; Kung et al., 2010). This characteristic allows bacteria to detect circumstantial osmotic changes more efficiency.

Two crystal structures of EcMscS (the MscS channel from Escherichia coli) have been solved. One is a wild-type EcMscS structure that presumably represents a closed state of MscS (Bass et al., 2002), and the other is EcMscS with an A106V mutation. This mutant has a substantial rotational rearrangement of the transmembrane (TM) region and an increased pore size; it is considered to reflect an open state of MscS (Wang et al., 2008). The MscS channels include two functional domains: a TM domain and a cytoplasmic domain. The TM domain enables the sensors to sense the tension exerted on the membrane or depolarization of the membrane potential (Bass et al., 2002; Bezanilla and Perozo, 2002). The cytoplasmic domain, with seven portals and a $\beta$-barrel (a distal pore at the bottom of $\mathrm{MscS}$ ) opening to the cytoplasm, is conserved in the MscS family (Perozo and Rees, 2003; Schumann et al., 2004). However, the function of the cytoplasmic domain is unclear. Recent biophysical studies have suggested that the cytoplasmic vestibule undergoes significant conformational changes when this channel opens (Miller et al., 2003). In addition, the cytoplasmic domain has been reported to be involved in the adaptation and ionselective process of the channel (Sotomayor et al., 2007; Gamini et al., 2011; Cox et al., 2013).

The crystal structure and functions of $T t \mathrm{MscS}$, an $\mathrm{MscS}$ channel from Thermoanaerobacter tengcongensis ( $T$. tengc), has recently been reported by our team (Zhang et al., 2012). Although, TtMscS has only $27 \%$ similarity to $E c \mathrm{MscS}$, their overall structural folds are similar. The entire structure of TtMscS closely resembles the $E c \mathrm{MscS}$ structure of the closed state except for two distinct cytoplasmic regions: the side portals and the bottom $\beta$-barrel. Sequence alignment combined with structural analysis revealed that three bulky residues, Phe157, Met243, and Trp246, lead to a much smaller portal for TtMscS than EcMscS. In contrast, the $\beta$-barrel in TtMscS is much larger than that in $E c M s c S$. In addition, the pattern of electrostatic surface potentials inside the $\beta$-barrel pore and around the $\beta$-barrel region between $T t \mathrm{MscS}$ and EcMscS are opposite. The larger pore size and distinctive surface potential distribution indicate that the $\beta$-barrel is a dominant pathway for ion entry in TtMscS (Zhang et al., 2012). However, the mechanistic details of the $\beta$-barrel structure to anion selection of $\mathrm{Tt} \mathrm{MscS}$ are still unclear.

In this study, on the basis of structural analysis, we introduced several mutations at key residues in the inner wall of the $\beta$-barrel pore, aiming to change the physical size and electrostatic potential of the side chains of these key residues. Our electrophysiological results showed that key point mutations in the $\beta$-barrel attenuated or reversed the anion selectivity of TtMscS. In conclusion, the present study defines several residues that are crucial for the anion selectivity of $T t \mathrm{MscS}$ and supports the hypothesis that the $\beta$-barrel of
$T t \mathrm{MscS}$ acts as a "selective filter" that confers TtMscS anion selectivity.

\section{MATERIALS AND METHODS}

\section{Protein Expression and Purification}

The gene encoding the MscS channel from T. tengcongensis (TtMscS) was cloned into pET-21, a vector with an N-terminal $6 \times$ His tag followed by a tobacco etch virus (TEV) protease cleavage site. The channel was expressed in BL21 cell cultures by induction with $0.4 \mathrm{mM}$ isopropyl- $\beta$-d-thiogalactopyranoside (IPTG) at $\mathrm{A}_{600} \sim 1.0$. The cells were cultured at $23^{\circ} \mathrm{C}$ for $18 \mathrm{~h}$ and collected. The collected bacteria were homogenized in lysis buffer (containing: $50 \mathrm{mM}$ Tris $\cdot \mathrm{HCl}$ and $200 \mathrm{mM} \mathrm{NaCl}$; $\mathrm{PH}: 8.0$ ) and lysed by sonication. The suspension was centrifuged $(15,000$ $\times \mathrm{g}$ ) for $15 \mathrm{~min}$ to remove cell debris. The supernatant was collected and centrifuged by ultracentrifugation $(100,000 \times \mathrm{g})$ for $1 \mathrm{~h}$ to obtain the membrane fraction (sediment). The membrane fraction was suspended in buffer A (containing $25 \mathrm{mM}$ Tris $\cdot \mathrm{HCl}$, $20 \mathrm{mM}$ imidazole and $500 \mathrm{mM} \mathrm{NaCl}$; PH: 8.0), and Triton X$100(0.5 \%, \mathrm{vol} / \mathrm{vol})$ and PMSF $(1 \mathrm{mM})$ were then added. The suspension was incubated at $4^{\circ} \mathrm{C}$ with slow stirring overnight. Then, the sample was subjected to ultracentrifugation $(100,000 \times$ g) for $30 \mathrm{~min}$. The supernatant was harvested and loaded onto a Ni-NTA column (GE Healthcare) pre-equilibrated with buffer A containing $0.1 \%$ Triton X-100. Next, the column was washed with buffer $A$ by adding $20 \mathrm{mM}$ imidazole and $0.02 \% \mathrm{n}$-dodecyl- $\beta$-Dmaltopyranoside (DDM) to detach any non-specific binding. The channel protein was washed with buffer B (containing $25 \mathrm{mM}$ Tris. $\mathrm{HCl}, 300 \mathrm{mM}$ imidazole, $500 \mathrm{mM} \mathrm{NaCl}$, and $0.02 \% \mathrm{DDM}$, $\mathrm{PH}: 8.0$ ), and the $\mathrm{N}$-terminal $6 \times$ His-tag was removed by TEV protease. The channel protein was concentrated and further purified by gel filtration (Superdex-200, GE Healthcare) with elution buffer ( $25 \mathrm{mM}$ Tris. $\mathrm{HCl}, 200 \mathrm{mM} \mathrm{NaCl}, 5 \mathrm{mM}$ DTT and $0.02 \%$ DDM; PH: 8.0). To confirm the purity of the protein, the peak fraction was collected and separated by SDS-PAGE and stained with Coomassie blue. The purified proteins were stored at $4^{\circ} \mathrm{C}$ for no more than 1 week for further functional studies.

\section{Preparation of Giant Liposomes and Electrical Recording}

All lipids used in this study were obtained from Avanti Polar Lipids. The wild-type TtMscS channel and mutant proteins were reconstituted into lipid vesicles composed of 1-pal-mitoyl-2oleoyl-phosphatidylethanolamine (POPE, $7.5 \mathrm{mg} / \mathrm{mL}$ ) and 1palmitoyl-2-oleoyl-phosphatidylglycerol (POPG, $2.5 \mathrm{mg} / \mathrm{mL}$ ) by using a previously described method (Li et al., 2007). To ensure single channel recording, the protein concentrations of lipids were all $150 \mu \mathrm{g} / \mathrm{ml}$. Before electrophysiological analysis, the lipids were subjected to regular dehydrate and hydrate processes to obtain giant liposomes. We performed patch-clamp recording in asymmetrical solution with conditioning in $150 \mathrm{mM}$ $\mathrm{KCl}, 500 \mathrm{mM}$ sucrose, $5 \mathrm{mM} \mathrm{K}$-HEPES ( $\mathrm{pH}$ 7.0) in the bath solution, and $15 \mathrm{mM} \mathrm{KCl,} 500 \mathrm{mM}$ sucrose, $5 \mathrm{mM} \mathrm{K-HEPES} \mathrm{(pH}$ 7.0 ) in the pipette solution (the 500 sucrose provides osmotic protection, prevents giant liposomes from bursting and stabilizes the gigaohm seal formation). When measuring the preference 
for other anions, $\mathrm{KCl}$ was exchanged to the corresponding saline solution $\left(\mathrm{KNO}_{3}, \mathrm{KF}, \mathrm{KBr}\right)$. Membrane voltages were controlled. After gigaohm seal (with a constant resistance of 3-8 G $\Omega$ ) formation, the current was recorded with an Axopatch 200B amplifier with a Digidata 1322A analog-to-digital converter (Axon Instruments). Single channel currents were recorded in Clampex 10.2 software, filtered at $2 \mathrm{kHz}$, and digitized at $5 \mathrm{kHz}$. The data were analyzed in Clampfit 10.2 software, and the current amplitudes were measured by the difference between the cursor aligned at the baseline and peak of the current traces. The mechanical pressure was measured with a pressure monitor (PM015D, WPI).

\section{Estimate of the Ion Conductance and Ion Permeability Ratio of TtMscS Channel}

We plotted single-channel currents against voltage to acquire $\mathrm{I}-\mathrm{V}$ curves. Ion conductance was estimated by the slope of the $\mathrm{I}-\mathrm{V}$ curves. The permeability ratios were calculated using the following versions of the Goldman-Hodgkin-Katz (GHK) equation:

$$
\mathrm{P}_{\mathrm{X}} / \mathrm{P}_{\mathrm{K}}=\frac{\left[K^{+}\right]_{0}-\left[K^{+}\right]_{i} \exp \left(-\frac{E_{\mathrm{Re} v} F}{R T}\right)}{\left[X^{-}\right]_{0} \exp \left(-\frac{E_{\mathrm{Re} v} F}{R T}\right)-\left[X^{-}\right]_{i}}
$$

where $\mathrm{X}$ is a monovalent anion. $[\mathrm{X}]_{\mathrm{o}} /\left[\mathrm{K}^{+}\right]_{\mathrm{o}}$ and $[\mathrm{X}]_{\mathrm{i}} /\left[\mathrm{K}^{+}\right]_{\mathrm{i}}$ are ion concentration on extracellular (cis-side) and intracellular sides (trans-side), respectively.

\section{Estimate of the Free Energy of Activation of the TtMscS Channel}

We estimated the free energy of activation of the TtMscS channel protein by using a previously described method (Kloda and Martinac, 2001). Briefly, the open probability of the $T t \mathrm{MscS}$ channel plotted against negative pressure was fitted to a Boltzmann distribution function given by:

$$
N P_{o}=N P_{\text {omax }}\left[1+\exp \alpha\left(p_{1 / 2}-p\right)\right]^{-1}
$$

where $N$ is the number of channels in the patch, and $\alpha$ is the sensitivity constant to negative pressure; $p$ is the negative pressure applied to the pipette, and $p_{1 / 2}$ is the negative pressure applied when the open probability of the channel is $0.5\left(P_{o}=0.5\right) . P_{o}$ and $P_{o}$ max represent the open probability and maximum open probability of the channel, respectively.

A two-state Boltzmann model was used to fit the gating of MS channels, with the change of area $t \Delta A$ as the dominant energy term (Sukharev et al., 1999). The free energy $\Delta G$ is a linear function of membrane tension, as shown in the following equation:

$$
\Delta G=t \Delta A-\Delta G_{o}
$$

where $\Delta G o$ is the difference in free energy between the closed and opened conformations of MS channels with the absence of the externally membrane tension. $\Delta A$ is the difference in membrane area occupied by open and closed channels at a given membrane tension, and $t \Delta A$ is the work for the gating of MS channels gated at the open probability of $0<P_{o}<1$.

Because the Boltzmann function for the open probability of MS channels can be written as

$$
P_{o} /\left(1-P_{o}\right)=\exp \left[\alpha\left(p-p_{1 / 2}\right)\right]=\exp \left[\left(t \Delta A-\Delta G_{o}\right) / k T\right] .
$$

By using Laplace's law

$$
t-t_{1 / 2}=\left(p-p_{1 / 2}\right)(r / 2) .
$$

where $r$ is the radius of curvature of the liposome patch shaped by the negative pressure applied to the pipette. Thus, when the open probability $\mathrm{P}_{\mathrm{o}}=0.5$ (i.e., $p=p_{1 / 2}$ and $t=t_{1 / 2}$ ) the free energy difference $\Delta G_{o}=0$. Consequently, $t_{1 / 2}=\Delta G o / \Delta A$ and $p_{1 / 2}=2 \Delta G_{o} / r \Delta A$, whereas $\alpha=r \Delta A / 2 k T$. The expression of the free energy for the activation of $T t \mathrm{MscS}$ channel in this study is given by

$$
\Gamma_{M S C}=\alpha P_{1 / 2}=\Delta G_{o} / k T \text {. }
$$

\section{Statistical Analyses}

All data presented in this paper were expressed as the mean \pm SEM. The ion selectivity $\left(\mathrm{P}_{\mathrm{Cl}} / \mathrm{P}_{\mathrm{K}}\right)$ was estimated by using the Goldman-Hodgkin-Katz $(\mathrm{GHK})$ equation. The $\mathrm{P}_{\mathrm{X}} / \mathrm{P}_{\mathrm{K}}$ ratios of $\mathrm{TtMscS}$ in different anion solutions (Figures 2C, 5C) were analyzed by using unpaired $t$-tests. The conductance (Figure 2D) and $\mathrm{P}_{\mathrm{Cl}} / \mathrm{P}_{\mathrm{K}}$ ratios of mutations (Figures $4 \mathrm{D}, 6 \mathrm{~B}, \mathrm{D}$ ) were analyzed using one-way ANOVA, and post-hoc LSD tests were performed.

\section{RESULTS}

\section{TtMscS Is a Mechano-Sensitive and Anion-Selective Channel}

We cloned the TtMscS gene into the pET-21a vector. The TtMscS channel was overexpressed in BL21 competent cells. Through gel filtration chromatography, we acquired high-purity TtMscS protein $(32 \mathrm{kDa}$ ) (Figure 1A). To detect the activity and physiological functions of TtMscS, we reconstituted highpurity protein into lipid vesicles (giant liposome) and tested the electrophysiological properties by using a patch-clamp recording system. In patch-clamp recording experiments, the $T t \mathrm{MscS}$ channel opened under negative pressure, thus reflecting a perfect mechanosensitive property (Figure 1B). The open probability plotted against negative pipette pressure was fitted to a Boltzmann distribution function (Figure 1D). The sensitivity parameter $(1 / \alpha)$ of $\mathrm{TtMscS}$ to pressure was $4.8 \pm 0.9 \mathrm{mmHg}$, and $\mathrm{P}_{1 / 2}$, the pressure at the point when the channel open probability was 0.5 , was $79.7 \pm 5.2 \mathrm{mmHg}$ (mean \pm SEM, $n=3$ ). Using $\Gamma_{M S C}=\alpha P_{1 / 2}=\Delta G_{o} / k T$, we obtained the free energy of the channel activation $\Delta G_{0}=17.5 \pm 2.9 \mathrm{kT}$ (see section Materials and Methods). The ion selectivity was measured with a reversal potential under asymmetric $\mathrm{KCl}$ solutions $(15 \mathrm{mM}$ $\mathrm{KCl}$ in pipette and $150 \mathrm{mM}$ in the bath). TtMscS exhibited an average reversal potential of $-26.7 \pm 0.9 \mathrm{mV}$ (Figure 1C, mean \pm SEM, $n=7)$. According to the GHK equation, we calculated the permeability ratio of TtMscS for chloride vs. potassium $\mathrm{P}_{\mathrm{Cl}} / \mathrm{P}_{\mathrm{K}}$ $\sim 4: 1$. The results suggested that $T t \mathrm{MscS}$ is a mechano-sensitive, anion-selective ion channel. 

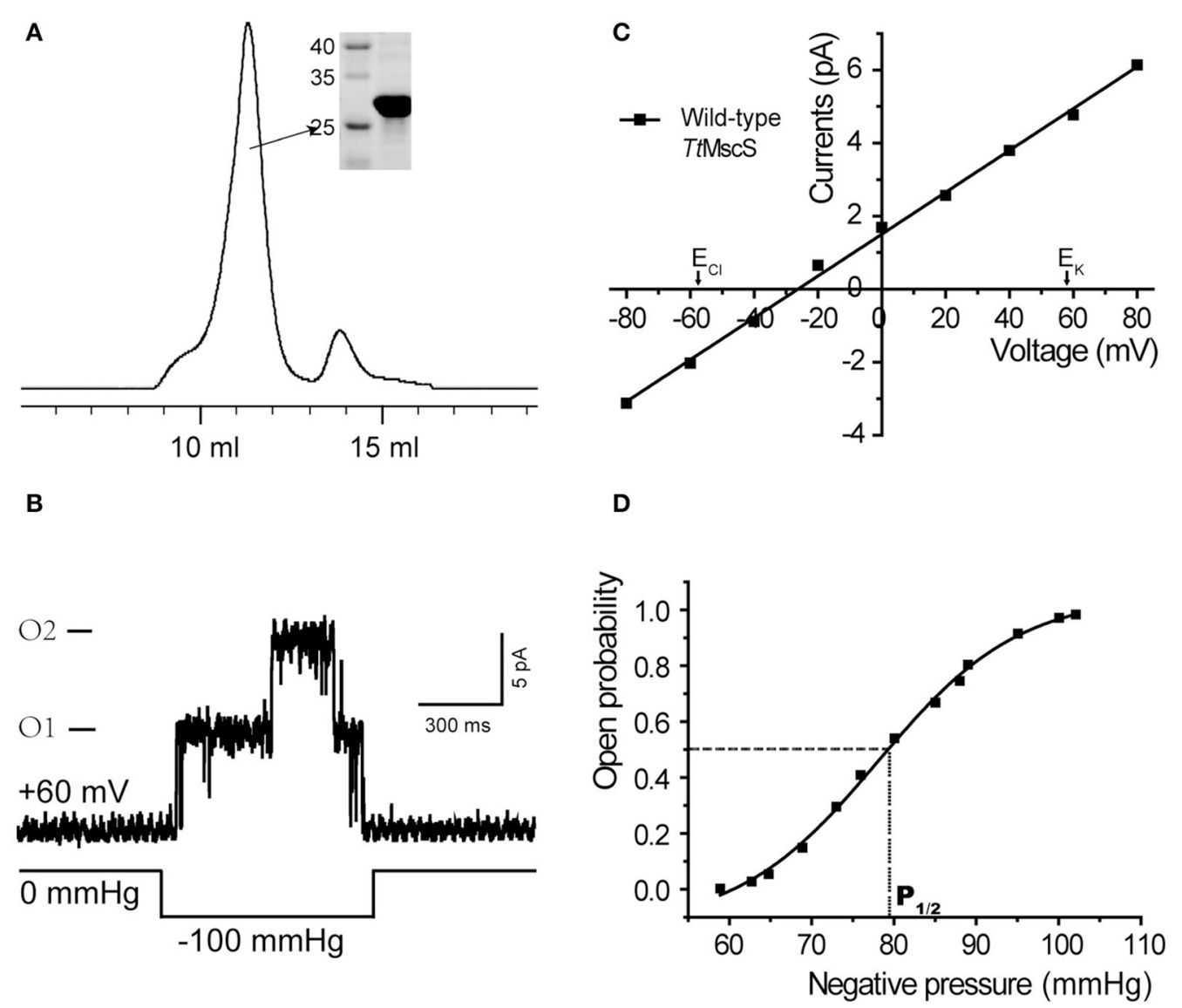

FIGURE 1 | TtMscS channels display strong mechano-sensitivity and anion selectivity. (A) Representative trace of TtMscS in gel filtration chromatography: volume to peak was 11-12 ml; purity of protein was identified by SDS-PAGE gel was stained with Coomassie blue. (B) Single-channel traces of TtMscS were recorded in asymmetric $\mathrm{KCl}$ solutions (bath/pipette: $150 / 15 \mathrm{mM}$ ) with a holding voltage of $+60 \mathrm{mV}$; single or dual channels opened under negative pressure applied to the patch pipette. (C) I-V curve for wild-type TtMscS recorded in asymmetric KCl solutions (bath/pipette: 150/15 mM). According to the GHK equation, the reversal potential of an ideal anion- or cation-selective channel was -58 or $+58 \mathrm{mV}$. The reversal potential for TtMscS was $-26.7 \pm 0.9 \mathrm{mV}$ (mean \pm SEM, $n=7)$. (D) Open probability of $T t$ MscS vs. negative pressure applied to the patch pipette fitted by the Boltzmann distribution function. $P_{1 / 2}$ represents negative pressure at the point at which the open probability is 0.5 .

\section{TtMscS Showed Different Degrees of Preference for Different Anions}

We demonstrated that $T t M s c S$ showed a preference for $\mathrm{Cl}^{-}$ over $\mathrm{K}^{+}$. Therefore, we further investigated the preference of $T t \mathrm{MscS}$ for other anions. To determine the anion preference of Tt MscS for various anions $\left(\mathrm{NO}_{3}^{-}, \mathrm{F}^{-}, \mathrm{Br}^{-}\right)$, we exchanged other anions $\left(\mathrm{NO}_{3}^{-}, \mathrm{F}^{-}, \mathrm{Br}^{-}\right)$in asymmetric solutions and contrasted the differences of corresponding reversal potentials. Our results demonstrated that $\mathrm{Tt} \mathrm{MscS}$ showed selective preferences for various anions, namely, $\mathrm{NO}_{3}^{-}>\mathrm{Cl}^{-} \approx \mathrm{F}^{-}>\mathrm{Br}^{-}$[Figures $2 \mathrm{~B}, \mathrm{C}$, Table 1, unpaired $t$-test, $t_{(1,10)}=5.047, p=0.001$ for $\mathrm{KNO}_{3}$ vs. $\mathrm{KCl}$ comparison; $t_{(1,10)}=2.906, p=0.2$ for $\mathrm{KCl}$ vs. $\mathrm{KF}$ comparison; $t_{(1,7)}=13.533, p=0.033$ for $\mathrm{KF}$ vs. $\mathrm{KBr}$ comparison]. Notably, Tt MscS showed much stronger selectivity for $\mathrm{NO}_{3}^{-}\left(\mathrm{E}_{\mathrm{rev}}=-48.6 \pm 1.9 \mathrm{mV}, \mathrm{P}_{\mathrm{Cl}} / \mathrm{P}_{\mathrm{K}} \sim 26: 1, n=5\right)$ than other anions, very similarly to the anion preference of the GABA receptor $\left(\mathrm{P}_{\mathrm{Na}} / \mathrm{P}_{\mathrm{K}} / \mathrm{P}_{\mathrm{Cl}} \sim 0: 0.03: 1\right.$; Wotring et al., 2003).
Ion conductance is a basic property of ion channels (Bezanilla, 2008). We compared the ion conductance of TtMscS performed with various anions $\left(\mathrm{NO}_{3}^{-}, \mathrm{Cl}^{-}, \mathrm{F}^{-}, \mathrm{Br}^{-}\right)$. Unexpectedly, TtMscS expressed much lower conductance to $\mathrm{F}^{-}$than other anions (including $\mathrm{Cl}^{-}, \mathrm{Br}^{-}$, and $\mathrm{NO}_{3}^{-}$) that are larger than $\mathrm{F}^{-}$ [Figures 2A,D, Table 1, one-way ANOVA, $F_{(3,17)}=14.069, p<$ 0.001 for Figure 2D]. This result suggested that the conductance of ion channels is determined not only by the physical size of the ions but also by the interaction between ions and ion channels. The lowest conductance for $\mathrm{F}^{-}$may be due to the large binding energy of $\mathrm{F}^{-}$with the TtMscS channel.

\section{$\beta$-Barrel Region May Confers the Anion Selectivity of TtMscS}

$\mathrm{Tt} \mathrm{MscS}$ is a homoheptameric complex, each monomer of which consists of 286 amino acids. Like other MscS channels, the TtMscS channel has two characteristic domains: a transmembrane domain and a cytoplasmic domain. The 

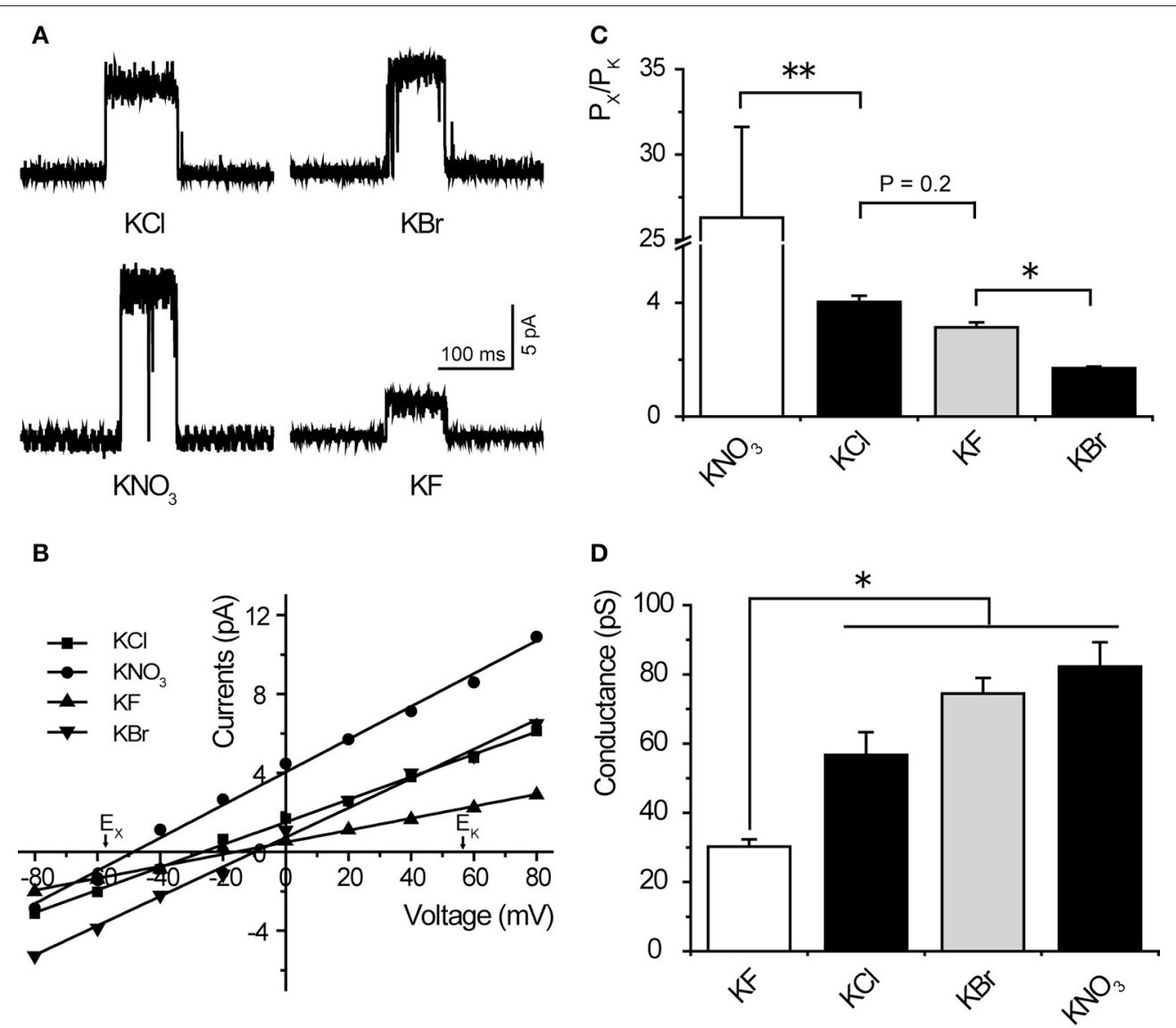

FIGURE 2 | TtMscS showed different preferences for different anions. (A) Single-channel traces of TtMscS in different anion solutions (KNO 3 , $\mathrm{KCl}$, $\mathrm{KF}$, $\mathrm{KBr}$ ) at +60 mV. (B) I-V curves for TtMscS recorded in asymmetric anion solutions ( $\mathrm{KNO}_{3}, \mathrm{KCl}, \mathrm{KF}, \mathrm{KBr}$ ) (bath/pipette: 150/15 mM). The reversal potentials were $-48.6 \pm 1.9 \mathrm{mV}$ (mean $\pm \mathrm{SEM}, n=5$ ), $-21.8 \pm 1.5 \mathrm{mV}$ (mean $\pm \mathrm{SEM}, n=5$ ), and $-11.8 \pm 1.0 \mathrm{mV}$ (mean $\pm \mathrm{SEM}, n=5)$ for $\mathrm{KNO} 3$, $\mathrm{KF}$ and $\mathrm{KBr}$, respectively. (C) lon selectivity $\left(\mathrm{P}_{\mathrm{Cl}} / \mathrm{P}_{\mathrm{K}}\right)$ of TtMscS in different asymmetric anion solutions $\left(\mathrm{KNO}_{3}, \mathrm{KCl}, \mathrm{KF}, \mathrm{KBr}\right)$. (D) lon conductance of TtMscS in different asymmetric anion solutions (KNO $\mathrm{K}_{3}, \mathrm{KCl}$, $\mathrm{KF}, \mathrm{KBr}) .{ }^{\star} p<0.05,{ }^{\star \star} p<0.01$.

cytoplasmic domain comprises two distinct openings: the portals and the $\beta$-barrel (Figures 3A,B). Despite the similar overall structural fold between $T t \mathrm{MscS}$ and $E c \mathrm{MscS}$, the portal and the $\beta$-barrel of these are strikingly different. Our previous results have indicated that the $\beta$-barrel may underlie the structural foundation for the anion-selective property of TtMscS (Zhang et al., 2012), but the mechanistic details by which the $\beta$-barrel determines anion selectivity are still vague. Therefore, we further analyzed the crystal structure of $T t \mathrm{MscS}$ and found three distinct residues-T272, V274, and L276-located in the inner wall of the $\beta$-barrel pore, and the V274 residue strikingly pointed at the center of the $\beta$-barrel (Figures 3C,D). On the basis of this structural information, we designed a series of mutations and tested their anion preferences by using a giant liposome patch-clamp system.

\section{Residue V274 Plays a Crucial Role in the Ion Selection of TtMscS}

Considering electrostatic potential, we mutated the V274 residue into a negatively charged aspartic acid (V274D) and tested it
TABLE 1 | Summary of properties of wild-type TtMscS in different anionic solutions.

\begin{tabular}{lrrcr}
\hline & $\mathbf{E}_{\text {rev }}(\mathbf{m V})$ & $\mathbf{P}_{\mathbf{X}} / \mathbf{P}_{\mathbf{K}}$ & Conductance $(\boldsymbol{\gamma}, \mathbf{p S})$ & $\boldsymbol{n}$ \\
\hline $\mathrm{KNO}_{3}$ & $-48.6 \pm 1.9$ & $26.3 \pm 5.3$ & $82.3 \pm 7.1$ & 5 \\
$\mathrm{KCl}$ & $-26.7 \pm 0.9$ & $4.0 \pm 0.2$ & $56.8 \pm 6.6$ & 7 \\
$\mathrm{KF}$ & $-21.8 \pm 0.5$ & $3.0 \pm 0.1$ & $30.2 \pm 2.1$ & 5 \\
$\mathrm{KBr}$ & $-11.8 \pm 1.0$ & $1.7 \pm 0.1$ & $74.5 \pm 4.5$ & 4 \\
\hline
\end{tabular}

by using the patch-clamp system in asymmetric $\mathrm{KCl}$ solutions (15 $\mathrm{mM} \mathrm{KCl}$ in pipette and $150 \mathrm{mM}$ in the bath). Surprisingly, the reversal potential shifted from $-26.7 \pm 0.9$ to $+11.3 \pm$ $1.1 \mathrm{mV}$ (mean \pm SEM, $n=4$ ), thus indicating that the V274D mutation reversed the anion selectivity of $T t \mathrm{MscS}$ to slight cation selectivity (Figures 4A,D). However, when we mutated the V274 residue to a positively charged arginine (V274R), the reversal potential did not exhibit a striking change compared with that of the V274D mutant (Figures 4B,D, shifted to $+7.9 \pm 2.9 \mathrm{mV}$, mean \pm SEM, $n=3$ ). We mutated V274 to alanine, which has 

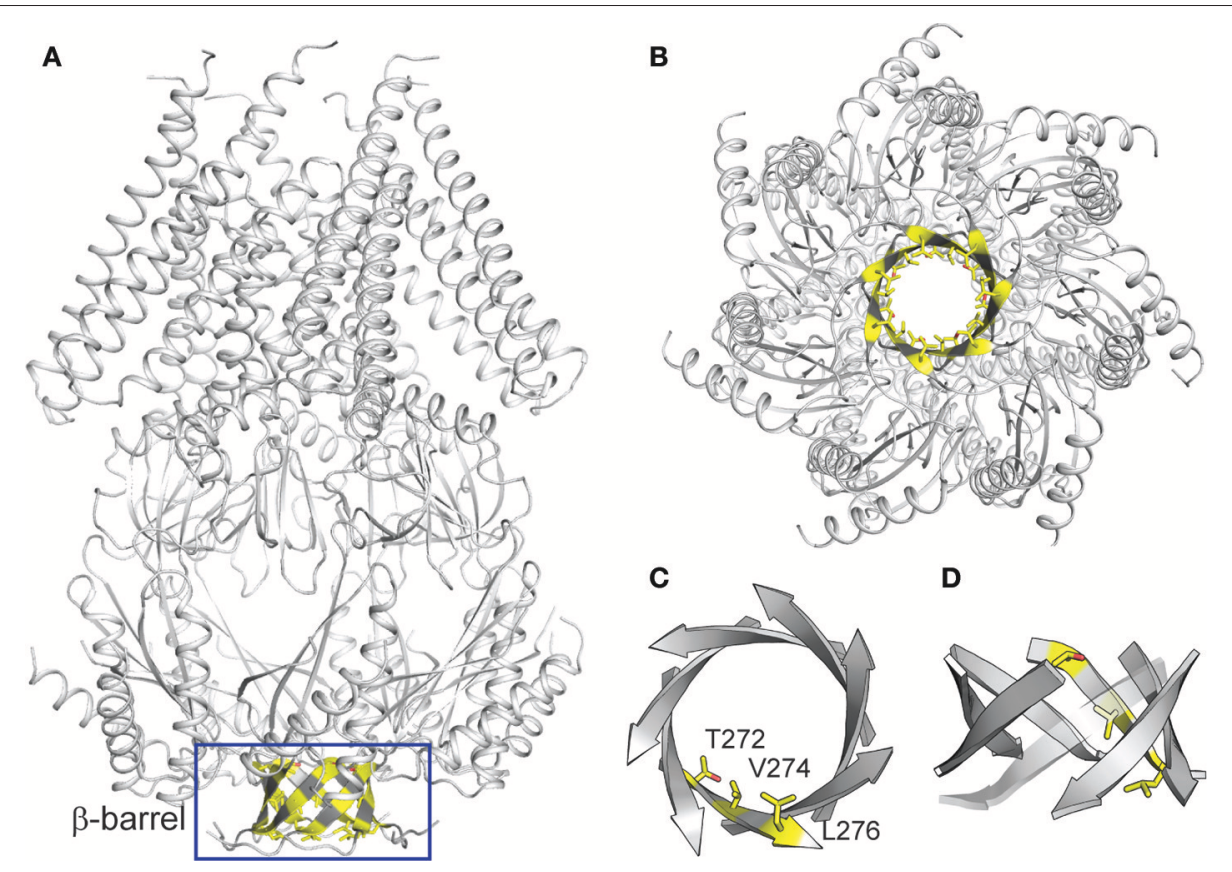

FIGURE 3 | Structural analysis of TtMscS. (A) overall structure of TtMscS homoheptamer with a $\beta$-barrel at the distal end of TtMscS. (B) Crystal structure of TtMscS viewed from the bottom, with residues T272, V274, and L276 in the inner wall of $\beta$-barrel shown as sticks. (C,D) Amplified structure of the $\beta$-barrel viewed from the bottom (C) and cytoplasmic sides (D). Three residues (T272, V274, L276) in one protomer are shown as sticks.

a smaller side chain (V274A). The V274A mutation shifted the reversal potential to $+15.4 \pm 2.0 \mathrm{mV}$ (Figures $4 \mathrm{C}, \mathrm{D}$, mean \pm SEM, $n=7$ ). Together, the three mutations at V274 neutralized the anion selectivity of TtMscS [Figure 4D, Table 2, one-way ANOVA, $\left.F_{(3,18)}=135.801, p<0.001\right]$ thus indicating that the V274 residue plays an essential role in the anion selectivity of TtMscS.

As shown in Figures 2B,C, wild-type TtMscS displayed much stronger selectivity for $\mathrm{NO}_{3}^{-}$than for the other monovalent anions $\left(\mathrm{Cl}^{-}, \mathrm{Br}^{-}\right.$, and $\left.\mathrm{F}^{-}\right)$. To determine whether $\mathrm{V} 274$ residue is also critical for the $\mathrm{NO}_{3}^{-}$selectivity of TtMscS channel, we further tested the reversal potential changes of V274A mutation in asymmetric $\mathrm{KNO}_{3}$ solutions. Similar to the situation in $\mathrm{KCl}$ solution, V274A reversed the reversal potential from $-48.6 \pm$ $1.9 \mathrm{mV}$ (wild type) to $+11.4 \pm 1.6$ (mean \pm SEM, $n=5$; V274A), which indicated that V274A mutation also neutralized the $\mathrm{NO}_{3}^{-}$ selectivity of TtMscS channel (Figures 5A-C, Table 3).

Collectivity, those results strongly support that V274 residue plays a vital role in the process of monovalent anions selectivity, including selectivity of $\mathrm{Cl}^{-}, \mathrm{NO}_{3}^{-}$, and maybe as well as $\mathrm{Br}^{-}, \mathrm{F}^{-}$, in TtMscS channel.

\section{The Electrostatic Potential of Residue T272 and the Physical Size of Residue L276 Affect the Anion Selectivity of TtMscS}

Our structural analysis showed that T272 and L276 are two additional residues located in the inner wall of the $\beta$-barrel. To identify the roles of these two residues in the anion selectivity of TtMscS, we mutated T272 and L276 to alanine (small physical size, T272A and L276A) or lysine (positively charged, T272K and L276K), respectively. The electrophysiological results showed that the reversal potential of the T272A mutant, compared with that of wild-type TtMscS, did not exhibit substantial changes (Figures 6A,B, Table 2, $\mathrm{E}_{\mathrm{rev}}=-23.7 \pm 0.7 \mathrm{mV}$, mean \pm SEM, $n=3$ ). However, the reversal potential of the T272K mutant shifted to $-9.9 \pm 1.4 \mathrm{mv}$ (mean $\pm \mathrm{SEM}, n=4$ ), thus indicating a significant attenuation of anion selection [Figures 6A,B, Table 2, one-way ANOVA, $F_{(2,11)}=37.443, p$ $<0.001$ for Figure 6C]. In addition, the results for the L276 mutant were opposite from those of the V274 mutant. L276A rather than $\mathrm{L} 276 \mathrm{~K}$ markedly changed the reversal potential [Figures 6C,D, Table 2, for L276A, $\mathrm{E}_{\mathrm{rev}}=-7.5 \pm 1.0 \mathrm{mV}$, mean \pm SEM, $n=4$; for $\mathrm{L} 276 \mathrm{~K}, \mathrm{E}_{\mathrm{rev}}=-25.0 \pm 1.2 \mathrm{mV}$, mean \pm SEM, $n=3$, one-way ANOVA, $F_{(2,11)}=37.881$, $p<0.001$ for Figure 6D]. From the above comparison, we concluded that the electrostatic potential of T272 and the physical size of L276 to notably influence the anion selection of TtMscS.

\section{DISCUSSION}

Ion selectivity is a basic property of ion channels and is crucial for their physiological function (Roux et al., 2011). In the present study, a single mutation and patch-clamp recording system were used to investigate the mechanism of anion selectivity of TtMscS. Our results suggested that $T t \mathrm{MscS}$ is a mechano-sensitive and anion-selective channel. The preference ratio was $\mathrm{P}_{\mathrm{Cl}} / \mathrm{P}_{\mathrm{K}} \sim$ $4: 1$, and the free energy of the channel activation was $\Delta \mathrm{G}_{\mathrm{o}}=$ 


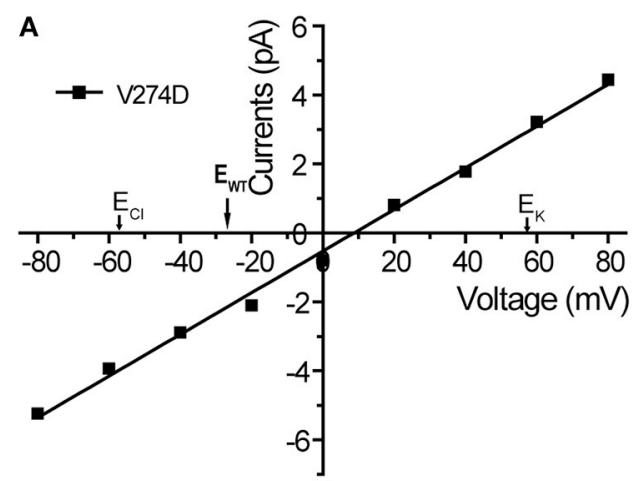

B

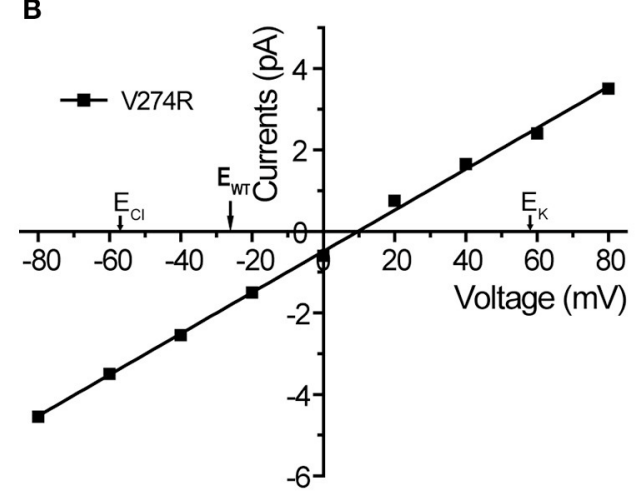

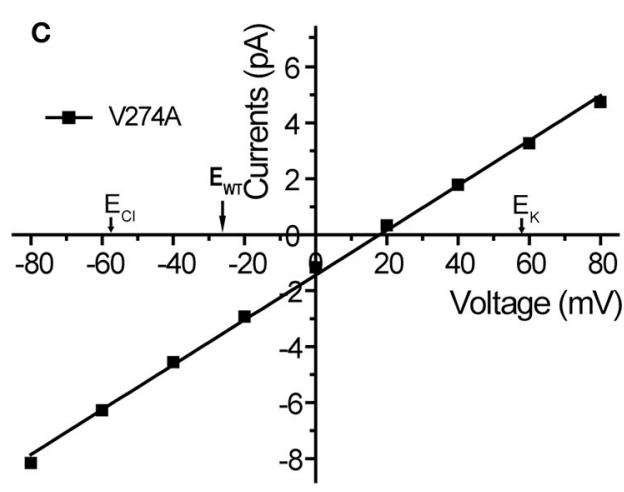

D

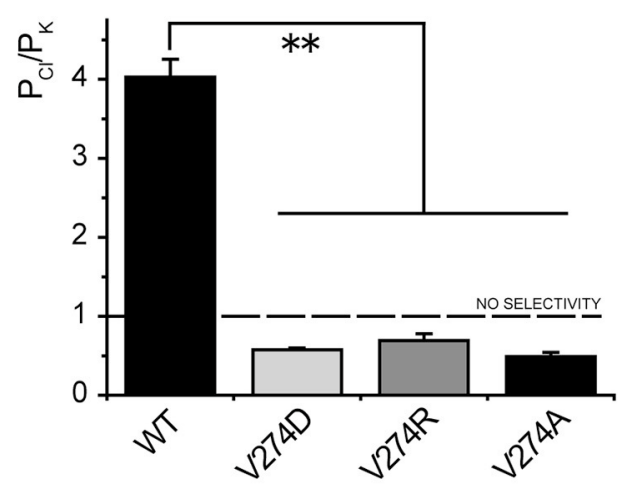

FIGURE 4 | Residue V274 inside the $\beta$-barrel plays a vital role in the anion selectivity of TtMscS. (A-C) I-V curves for mutations V274D, V274R, and V274A. The reversal potentials were $+11.3 \pm 1.1 \mathrm{mV}$ (mean $\pm \mathrm{SEM}, n=4),+7.9 \pm 2.9 \mathrm{mV}$ (mean $\pm \mathrm{SEM}, n=3)$, and $+15.4 \pm 2.0 \mathrm{mV}(\mathrm{mean} \pm \mathrm{SEM}, n=7)$ for V274D, V274R, and V274A, respectively. The arrow $\left(E_{W T}\right)$ represents the reversal potential position of wild-type TtMscS. (D) Summary of ion selectivity ( $\left.P_{C l} / P_{K}\right)$ for wild-type TtMscS (WT), V274D, V274R, and V274A mutations. ${ }^{* *} p<0.01$.

TABLE 2 | Summary of properties of wild-type TtMscS (WT) and mutants.

\begin{tabular}{lcccr}
\hline & E $r$ rev $(\mathbf{m V})$ & $\mathbf{P}_{\mathbf{C l}} / \mathbf{P}_{\mathbf{K}}$ & Conductance $(\boldsymbol{\gamma}, \mathbf{p S})$ & $\boldsymbol{n}$ \\
\hline WT & $-26.7 \pm 0.9$ & $4.0 \pm 0.2$ & $56.8 \pm 6.6$ & 7 \\
V274D & $+11.3 \pm 1.1$ & $0.6 \pm 0.1$ & $64.8 \pm 9.2$ & 4 \\
V274R & $+7.9 \pm 2.9$ & $0.7 \pm 0.1$ & $77.3 \pm 28.2$ & 3 \\
V274A & $+15.4 \pm 2.0$ & $0.5 \pm 0.1$ & $75.4 \pm 19.5$ & 7 \\
T272A & $-23.7 \pm 0.7$ & $3.5 \pm 0.1$ & $53 \pm 8.2$ & 3 \\
T272K & $-9.9 \pm 1.4$ & $1.6 \pm 0.1$ & $69.8 \pm 5.4$ & 4 \\
L276A & $-7.5 \pm 1.0$ & $1.5 \pm 0.1$ & $72.5 \pm 14.1$ & 4 \\
L276K & $-25 \pm 1.2$ & $3.7 \pm 0.3$ & $54.7 \pm 14.2$ & 3 \\
\hline
\end{tabular}

$17.5 \pm 2.9 \mathrm{kT}$. Second, TtMscS showed varying preferences for different anions (the degree order was $\mathrm{NO}_{3}^{-}>\mathrm{Cl}^{-} \approx \mathrm{F}^{-}>$ $\mathrm{Br}^{-}$), especially for $\mathrm{NO}_{3}^{-}$, for which $T t \mathrm{MscS}$ exhibited a more marked preference as compared with other anions $\left(\mathrm{E}_{\mathrm{rev}}=-48.6\right.$ $\left.\pm 1.9, \mathrm{P}_{\mathrm{NO} 3} / \mathrm{P}_{\mathrm{K}} \sim 26: 1\right)$. A conductance comparison suggested that $\mathrm{TtMscS}$ had much lower conductance to $\mathrm{F}^{-}$than to other anions $\left(\mathrm{Cl}^{-}, \mathrm{Br}^{-}, \mathrm{NO}_{3}^{-}\right)$larger than $\mathrm{F}^{-}$. Third, mutations at the center of the $\beta$-barrel (V274) reversed the reversal potentials and thus reversed the ion preference (from anion preference to cation preference) of $\mathrm{Tt} \mathrm{MscS}$. Finally, changes in electrostatic potential (T272K) and physical size (L276A) of residues in the inner wall of $\beta$-barrel both attenuated the anion preference of $T t$ MscS. Together, our results indicated that all central pointed residues located in the inner wall of the $\beta$-barrel play a crucial role in the anion selectivity of TtMscS. Generally, functional properties are based on structural domains. The present results suggested that the $\beta$-barrel of TtMscS acts as a "selective filter" that confers Tt MscS its anion selection.

Mechanisms underlying the anion selectivity of ion channels have been reported previously. However, the location of selective filters varies substantially among anion selective channels. $\mathrm{ClC}$ chloride channels belong to a large $\mathrm{Cl}^{-}$channel family found in organisms including bacteria and animals (Dutzler, 2006). In addition to $\mathrm{Cl}^{-}, \mathrm{ClC}$ chloride channels are selective for other small monovalent anions $\left(\mathrm{Br}^{-}, \mathrm{I}^{-}, \mathrm{NO}_{3}^{-}\right.$, and $\mathrm{SCN}^{-}$; Miller, 2006). Structural studies have indicated that an appropriate hourglass-shaped ion pathway and a partial positive binding site together determine the anion selectivity of $\mathrm{ClC}$ channels (Dutzler et al., 2002, 2003; Dutzler, 2007). This finding might describe principles of anion selectivity for anion-selective ion channels, despite differences between ion channels and pumps. In mammals, GABA receptors are ligand-gated chloride channels, which are inhibitory neurotransmitter receptors critical for maintaining appropriate neuronal activity and synaptic transmission (Wotring et al., 2003; Sigel and Steinmann, 2012). 

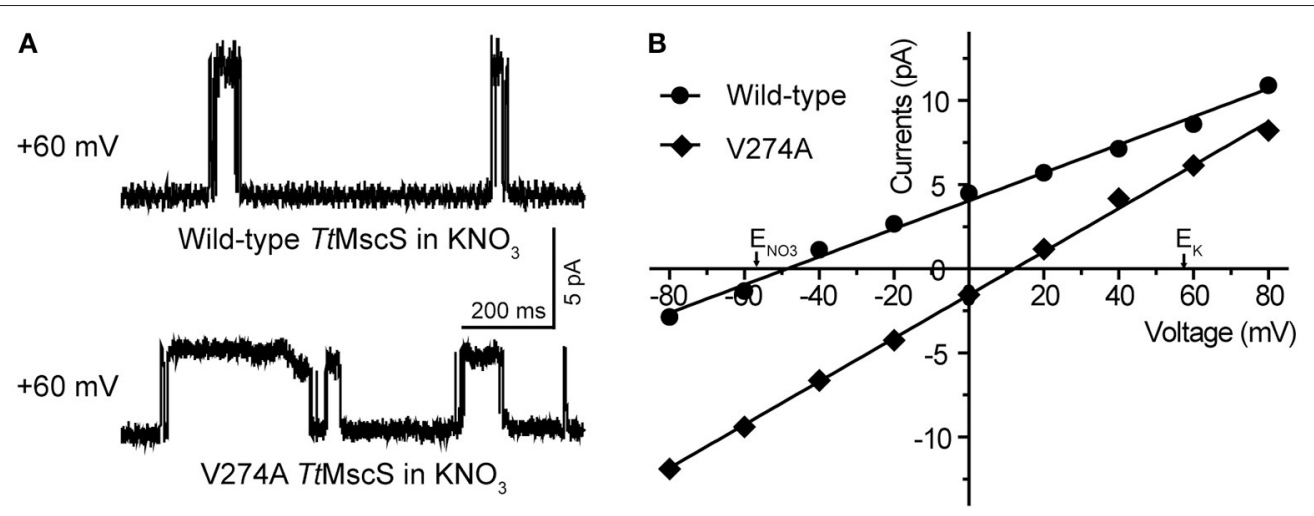

C

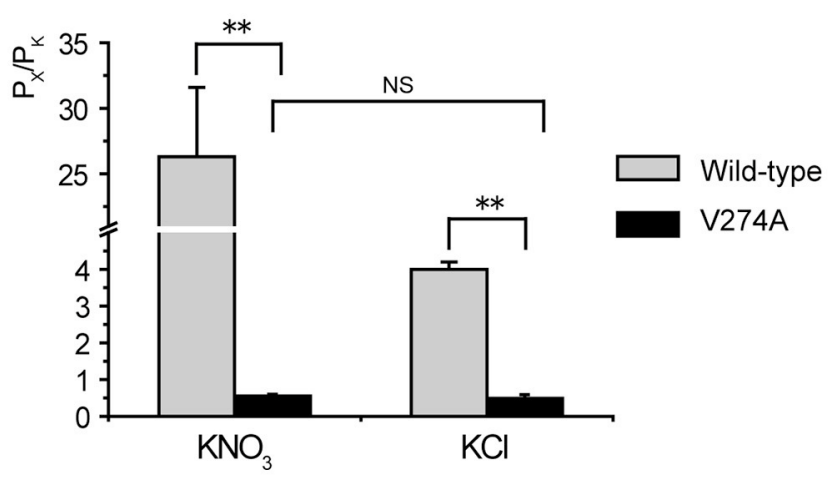

FIGURE 5 | V274 residue is critical for the $\mathrm{NO}_{3}^{-}$selectivity of TtMscS channel. (A) Single-channel traces of wild-type TtMscS and V274A mutation in $\mathrm{KNO}_{3}$ solution at $+60 \mathrm{mV}$. (B) I-V curves for TtMscS and V274A mutation recorded at asymmetric $\mathrm{KNO}_{3}$ solution. The reversal potentials of V274A mutation was $+11.4 \pm 1.6 \mathrm{mV}$ (mean $\pm \mathrm{SEM}, n=5$ ). (C) The summary of ion selectivity $\left(\mathrm{Px}_{\mathrm{X}} / \mathrm{P}_{\mathrm{K}}: \mathrm{P}_{\mathrm{NO} 3} / \mathrm{PK}_{\mathrm{K}}\right.$ or $\left.\mathrm{P}_{\mathrm{Cl}} / \mathrm{PK}_{\mathrm{K}}\right)$ for wild-type TtMscS and V274A mutation in $\mathrm{KNO}_{3}$ and $\mathrm{KCl}$ solutions. NS: no significant difference. ${ }^{*} p<0.01$.

TABLE 3 | Property comparison of wild-type TtMscS (WT) and V274A mutant in $\mathrm{KNO}_{3}$ and $\mathrm{KCl}$ solutions.

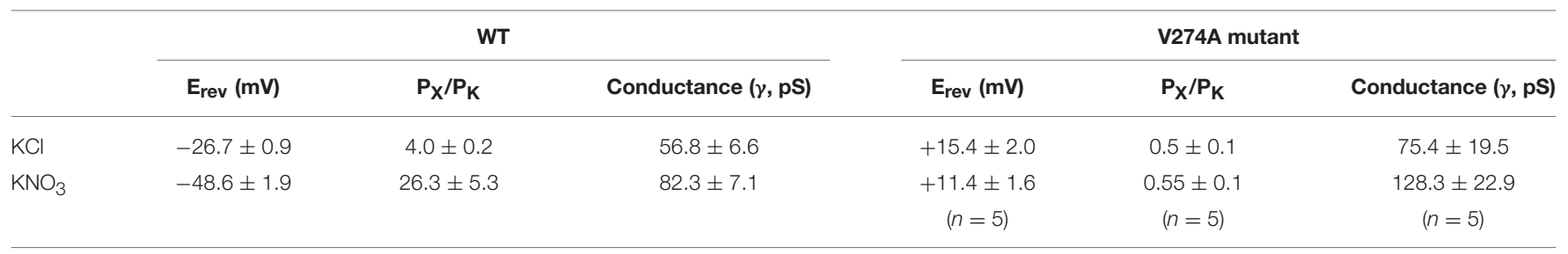

However, the mechanism underlying the chloride selectivity of GABA receptors is unclear. Glutamate-gated chloride channel $\alpha(\mathrm{GluCl})$ is a glutamate-gated chloride selective channel that belongs to the same family of GABA receptors (Cys-loop family). A structural analysis has demonstrated that the electropositive concave pocket at the bottom of $\mathrm{GluCl}$ channel is crucial for the anion selective property (Hibbs and Gouaux, 2011).

Unlike voltage-gated $\mathrm{K}^{+}, \mathrm{Na}^{+}, \mathrm{Ca}^{2+}$, and $\mathrm{Cl}^{-}$channels, whose selectivity arises from specific residues in the TM domain, the selectivity of MscS channels is defined by the physical size and charge distribution within the cytoplasmic vestibular domain (Gamini et al., 2011). Quantitative studies have reported that the cytoplasmic equatorial portals act as selective-filters and gateways for ion permeation in MscS channels (Perozo and Rees, 2003; Martinac et al., 2008; Gamini et al., 2011; Cox et al.,
2013). However, the structural comparison showed that TtMscS has smaller portals but a larger $\beta$-barrel than EcMscS (Zhang et al., 2012). In addition, the size and charge distribution of the cylindrical $\beta$-barrel pore in TtMscS is highly similar to the bottom electropositive concave pocket of the $\mathrm{GluCl}$ channel (Hibbs and Gouaux, 2011; Zhang et al., 2012). Moreover, the $\beta$ barrel is a common structural motif in outer membrane proteins (OMPs) which have been described in the outer membranes of bacteria (Schulz, 2002) and mitochondria (Heins et al., 1994; Hoogenboom et al., 2007). Voltage-gated anion channels (VDAC), which are outer membrane proteins of mitochondria characterized by a transmembrane $\beta$-barrel structure, show a slight anion selectivity (Heins et al., 1994). All of these findings suggest that the $\beta$-barrel of TtMscS might reasonably be capable of ion permeation and ion selection. In the present study, a 

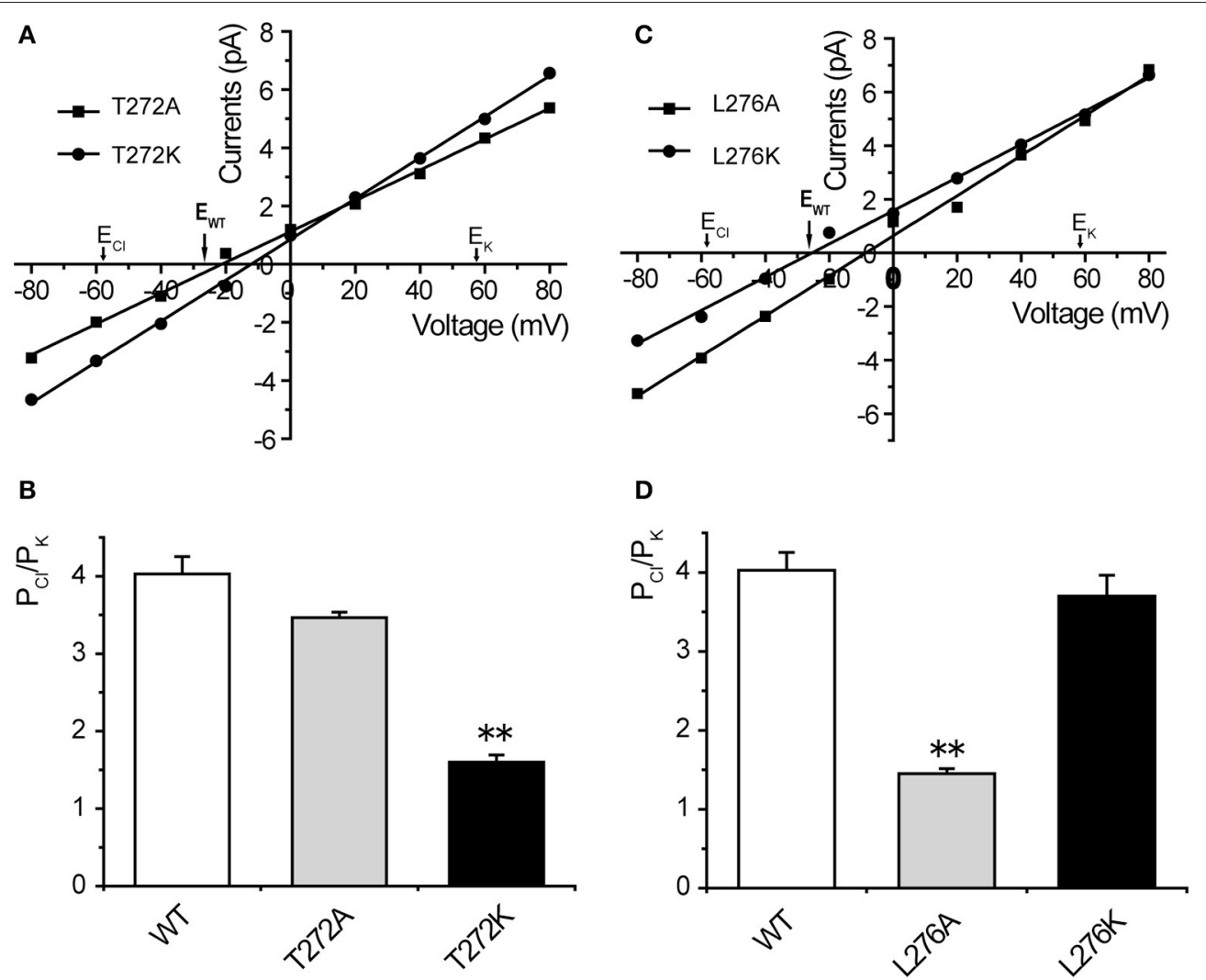

FIGURE 6 | T272 and L276 residues co-determined the anion selectivity of TtMscS. (A) I-V curves for T272A and T272K. The reversal potentials were $-23.7 \pm$ $0.7 \mathrm{mV}$ (mean $\pm \mathrm{SEM}, n=3$ ) and $-9.9 \pm 1.4 \mathrm{mV}$ (mean $\pm \mathrm{SEM}, n=4$ ) for T272A and T272K, respectively. The arrow (EWT) indicates the reversal potential position of wild-type TtMscS. (B) Summary of ion selectivity ( $\left.\mathrm{P}_{\mathrm{Cl}} / \mathrm{P}_{\mathrm{K}}\right)$ for wild-type TtMscS (WT), T272A, and T272K mutations. (C) I-V curves for L276A and L276K. The reversal potentials were $-7.5 \pm 1.0 \mathrm{mV}$ (mean $\pm \mathrm{SEM}, n=4$ ) and $-25.0 \pm 1.2 \mathrm{mV}$ (mean $\pm \mathrm{SEM}, n=3$ ) for L276A and L276K, respectively. The arrow (EWT) indicates the reversal potential of wild-type TtMscS. (D) Summary of ion selectivity $\left(\mathrm{P}_{\mathrm{Cl}} / \mathrm{P}_{\mathrm{K}}\right)$ for wild-type TtMscS (WT), L276A and L276K mutations. ${ }^{* \star} p<0.01$.

single residue mutation at the center of the $\beta$-barrel (V274) neutralized and even reversed the anion selectivity of TtMscS, thus suggesting a crucial role for residue locates at the center of $\beta$-barrel for the anion selectivity of TtMscS. Mutations of the other two extrusive residues at the inner wall of the $\beta$-barrel both attenuated anion selectivity, thus further verifying the crucial role of the $\beta$-barrel in the anion selectivity. The presented results suggest that the $\beta$-barrel acts as a "selective filter" module at the distal of anion selection of TtMscS. Complex proteins comprise a series of functional domains that endow the characteristic properties of $T t \mathrm{MscS}$ and confers this channel with a moderate degree of anion selectivity.

The MS channel responds to mechanical tension along the plane of the membrane. Studies have shown that the movement of transmembrane helices plays a critical role in the gating process of MscS channels (Martinac, 2004; Sotomayor and Schulten, 2004), suggesting that TM1 and TM2 may be responsible for gating the channel (Böttcher et al., 2015). In addition, the interaction of charged and polar residues in the TM1-TM2 helices with lipid headgroups can also affect the gating of MS channels (Sotomayor and Schulten, 2004). In the present study, the results showed that the TtMscS channel was open under negative pressure, but the negative pressure corresponding to the $1 / 2$ channel open probability $\left(\mathrm{P}_{1 / 2}\right)$ of $T t \mathrm{MscS}$ was much larger than that for $E c M s c S$ ( -79.7 vs. $-36 \mathrm{mmHg}$ ), even though both proteins belong to the same family and have very similar structures. The different pressure sensibilities between TtmscS and $E c M s c S$ may arise from the distinct arrangement of amino acid residues in the TM domain.

In summary, our results provide new insight into the molecular mechanism of the ion permeability and selectivity of the TtMscS channel. Our results indicate that both the electrostatic potential and the physical size of side chains inside the "selective filter" determine the anion selectivity of TtMscS, and thus may aid in understanding the mechanism of substrate selectivity of OMPs and the anion selectivity of chloride channels in eukaryotes.

\section{AUTHOR CONTRIBUTIONS}

YS, BZ, and FG: Performed all the experiments. BZ, MY, and YL: Initiated the project. All the authors contributed to data analysis and paper writing. 


\section{FUNDING}

This work was supported by the National Natural Science Foundation (31200771 to FG, 31171011, 31371066, 31671049 to YL), the Ministry of Science and Technology (2013CB91060101

\section{REFERENCES}

Arnadóttir, J., and Chalfie, M. (2010). Eukaryotic mechanosensitive channels. Annu. Rev. Biophys. 39, 111-137. doi: 10.1146/annurev.biophys.37.032807.125836

Bass, R. B., Strop, P., Barclay, M., and Rees, D. C. (2002). Crystal structure of Escherichia coli MscS, a voltage-modulated and mechanosensitive channel. Science 298, 1582-1587. doi: 10.1126/science.1077945

Bezanilla, F. (2008). Ion channels: from conductance to structure. Neuron 60 , 456-468. doi: 10.1016/j.neuron.2008.10.035

Bezanilla, F., and Perozo, E. (2002). Structural biology. Force and voltage sensors in one structure. Science 298, 1562-1563. doi: 10.1126/science.1079369

Böttcher, B., Prazak, V., Rasmussen, A., Black, S. S., and Rasmussen, T. (2015). The structure of YnaI implies structural and mechanistic conservation in the MscS family of mechanosensitive channels. Structure 23, 1705-1714. doi: 10.1016/j.str.2015.06.023

Cox, C. D., Nomura, T., Ziegler, C. S., Campbell, A. K., Wann, K. T., and Martinac, B. (2013). Selectivity mechanism of the mechanosensitive channel MscS revealed by probing channel subconducting states. Nat. Commun. 4, 2137. doi: $10.1038 /$ ncomms 3137

Dutzler, R. (2006). The ClC family of chloride channels and transporters. Curr. Opin. Struct. Biol. 16, 439-446. doi: 10.1016/j.sbi.2006.06.002

Dutzler, R. (2007). A structural perspective on $\mathrm{ClC}$ channel and transporter function. FEBS Lett. 581, 2839-2844. doi: 10.1016/j.febslet.2007.04.016

Dutzler, R., Campbell, E. B., and MacKinnon, R. (2003). Gating the selectivity filter in ClC chloride channels. Science 300, 108-112. doi: 10.1126/science. 1082708

Dutzler, R., Campbell, E. B., Cadene, M., Chait, B. T., and MacKinnon, R. (2002). $\mathrm{X}$-ray structure of a $\mathrm{ClC}$ chloride channel at $3.0 \mathrm{~A}$ reveals the molecular basis of anion selectivity. Nature 415, 287-294. doi: 10.1038/415287a

Gamini, R., Sotomayor, M., Chipot, C., and Schulten, K. (2011). Cytoplasmic domain filter function in the mechanosensitive channel of small conductance. Biophys. J. 101, 80-89. doi: 10.1016/j.bpj.2011.05.042

Heins, L., Mentzel, H., Schmid, A., Benz, R., and Schmitz, U. K. (1994). Biochemical, molecular, and functional characterization of porin isoforms from potato mitochondria. J. Biol. Chem. 269, 26402-26410.

Hibbs, R. E., and Gouaux, E. (2011). Principles of activation and permeation in an anion-selective Cys-loop receptor. Nature 474, 54-60. doi: 10.1038/nature10139

Hoogenboom, B. W., Suda, K., Engel, A., and Fotiadis, D. (2007). The supramolecular assemblies of voltage-dependent anion channels in the native membrane. J. Mol. Biol. 370, 246-255. doi: 10.1016/j.jmb.2007.04.073

Kloda, A., and Martinac, B. (2001). Structural and functional differences between two homologous mechanosensitive channels of Methanococcus jannaschii. EMBO J. 20, 1888-1896. doi: 10.1093/emboj/20.8.1888

Kung, C. (2005). A possible unifying principle for mechanosensation. Nature 436, 647-654. doi: 10.1038/nature03896

Kung, C., Martinac, B., and Sukharev, S. (2010). Mechanosensitive channels in microbes. Annu. Rev. Microbiol. 64, 313-329. doi: 10.1146/annurev.micro.112408.134106

Li, Y., Berke, I., Chen, L., and Jiang, Y. (2007). Gating and inward rectifying properties of the MthK K+ channel with and without the gating ring. J. Gen. Physiol. 129, 109-120. doi: 10.1085/jgp.200609655

Martinac, B. (2004). Mechanosensitive ion channels: molecules of mechanotransduction. J. Cell Sci. 117, 2449-2460. doi: 10.1242/jcs.01232

Martinac, B., Buechner, M., Delcour, A. H., Adler, J., and Kung, C. (1987). Pressuresensitive ion channel in Escherichia coli. Proc. Natl. Acad. Sci. U.S.A. 84, 2297-2301. doi: 10.1073/pnas.84.8.2297 to YL), the Science and Technology Commission of Shanghai Municipality (16411967400 to ZL), and the "Personalized Medicines-Molecular Signature-based Drug Discovery and Development," Strategic Priority Research Program of the Chinese Academy of Sciences, (Grant No. XDA12040214).
Martinac, B., Saimi, Y., and Kung, C. (2008). Ion channels in microbes. Physiol. Rev. 88, 1449-1490. doi: 10.1152/physrev.00005.2008

Miller, C. (2006). ClC chloride channels viewed through a transporter lens. Nature 440, 484-489. doi: 10.1038/nature04713

Miller, S., Bartlett, W., Chandrasekaran, S., Simpson, S., Edwards, M., and Booth, I. R. (2003). Domain organization of the MscS mechanosensitive channel of Escherichia coli. EMBO J. 22, 36-46. doi: 10.1093/emboj/ cdg011

Perozo, E., and Rees, D. C. (2003). Structure and mechanism in prokaryotic mechanosensitive channels. Curr. Opin. Struct. Biol. 13, 432-442. doi: 10.1016/S0959-440X(03)00106-4

Ranade, S. S., Syeda, R., and Patapoutian, A. (2015). Mechanically activated ion channels. Neuron 87, 1162-1179. doi: 10.1016/j.neuron.2015.08.032

Roux, B., Berneche, S., Egwolf, B., Lev, B., Noskov, S. Y., Rowley, C. N., et al. (2011). Ion selectivity in channels and transporters. J. Gen. Physiol. 137, 415-426. doi: 10.1085/jgp.201010577

Schulz, G. E. (2002). The structure of bacterial outer membrane proteins. Biochim. Biophys. Acta 1565, 308-317. doi: 10.1016/S0005-2736(02)00577-1

Schumann, U., Edwards, M. D., Li, C., and Booth, I. R. (2004). The conserved carboxy-terminus of the MscS mechanosensitive channel is not essential but increases stability and activity. FEBS Lett. 572, 233-237. doi: 10.1016/j.febslet.2004.07.045

Sigel, E., and Steinmann, M. E. (2012). Structure, function, and modulation of GABA(A) receptors. J. Biol. Chem. 287, 40224-40231. doi: 10.1074/jbc.R112.386664

Sotomayor, M., and Schulten, K. (2004). Molecular dynamics study of gating in the mechanosensitive channel of small conductance MscS. Biophys. J. 87, 3050-3065. doi: 10.1529/biophysj.104.046045

Sotomayor, M., Vasquez, V., Perozo, E., and Schulten, K. (2007). Ion conduction through MscS as determined by electrophysiology and simulation. Biophys. J. 92, 886-902. doi: 10.1529/biophysj.106.095232

Sukharev, S. I., Sigurdson, W. J., Kung, C., and Sachs, F. (1999). Energetic and spatial parameters for gating of the bacterial large conductance mechanosensitive channel, MscL. J. Gen. Physiol. 113, 525-540. doi: 10.1085/jgp.113.4.525

Wang, W., Black, S. S., Edwards, M. D., Miller, S., Morrison, E. L., Bartlett, W., et al. (2008). The structure of an open form of an E. coli mechanosensitive channel at 3.45 A resolution. Science 321, 1179-1183. doi: 10.1126/science.11 59262

Wotring, V. E., Miller, T. S., and Weiss, D. S. (2003). Mutations at the GABA receptor selectivity filter: a possible role for effective charges. J. Physiol. 548(Pt 2), 527-540. doi: 10.1113/jphysiol.2002.032045

Zhang, X., Wang, J., Feng, Y., Ge, J., Li, W., Sun, W., et al. (2012). Structure and molecular mechanism of an anion-selective mechanosensitive channel of small conductance. Proc. Natl. Acad. Sci. U.S.A. 109, 18180-18185. doi: $10.1073 /$ pnas. 1207977109

Conflict of Interest Statement: The authors declare that the research was conducted in the absence of any commercial or financial relationships that could be construed as a potential conflict of interest.

Copyright (c) 2017 Song, Zhang, Guo, Yang, Li and Liu. This is an open-access article distributed under the terms of the Creative Commons Attribution License (CC BY). The use, distribution or reproduction in other forums is permitted, provided the original author(s) or licensor are credited and that the original publication in this journal is cited, in accordance with accepted academic practice. No use, distribution or reproduction is permitted which does not comply with these terms. 